\title{
Jährliches Treffen der Arbeitskreis-Leiter und des Vorstands
}

Wie jedes Jahr fand am 2.4.2016 diesmal wieder in Elixhausen/Salzburg das gemeinsame Treffen der AK-Leiter mit dem Vorstand statt, um die Arbeitskreise über Vereins-Entwicklungen zu informieren, aber auch den schnellen und offenen Informationsaustausch untereinander zu pflegen. Acht verschiedene Arbeitskreise haben mit dem Vorstand anliegende Fragen in einer wie gewohnt sehr entspannten Atmosphäre diskutiert. Das vollständige Protokoll findet sich nach dem MemberLogin auf der Webpage (www.oegum.at); hier nur einige Auszüge daraus (CK):

\section{Erweiterte Vorstandssitzung der ÖGUM (Auszug) \\ $\nabla$}

in Elixhausen/Salzburg am 2.4.2016 mit ca. 20 Vertretern von Arbeitskreisen und des Vorstands, vertretene Arbeitskreise: Abdomen / Allgemeinmedizin / Gynäkologie / Geburtshilfe / Kopf / Hals / Mammasonographie / Notfallsonographie / Technik/Thorax; andere AKs ließen sich entschuldigen.

\section{Genehmigung des Protokolls der} Erweiterten Vorstandssitzung vom 30.05.2015

A. Klauser bittet, im Protokoll der letztjährigen erweiterten Vorstandssitzung unter den Stufe III-Voraussetzungen, die mindestens 5-jährige aktive Ultraschalldiagnostik zu vermerken und nicht wie angegeben, 6-jährige.

T. Ybinger weist auf die zwei Jahre hin, die zwischen Stufe I und II liegen sollte. Weisen mit diesem Vorgehen Interessenten $\mathrm{ab}$, die sich gerne zertifizieren möchten.

C. Weismann schließt sich dieser Meinung an. A. Klauser fasst zusammen, dass eine Ausnahmeregelung in Einzelfällen möglich ist.

\section{Vorstellung Firma Mides}

A. Klauser begrüßt Herrn Dutzi von der Firma Mides, welche eine Partnerschaft mit der ÖGUM anbietet und Kurse bzw. Seminare unterstützen kann, dies auch hinsichtlich des DLT 2017 in Linz.

\section{Pläne / Neuigkeiten der Arbeits- kreise:}

Mammasonographie: C. Weismann berichtet über durchgeführte Kurse, Vorträge, Skripten, Tutor- und Kursleitungen. M. Daniaux hat ebenfalls mit Kurstätigkeit erfolgreich begonnen, weitere Referententätigkeit bspw. bei Dreiländertreffen, Vorträge auch bei Gyn. Ultraschallkongressen. Enger Kontakt mit DEGUM. Wahrscheinlich muss man bei dem Thema Grundkurse nachjustieren. Über zwei Tage oft schwierig, sinnvoller sind 1-Tageskurse, die man deshalb durchaus auch modular organisieren kann. Üblicherweise auf einen Tag mit 10 DFP Punkten und 8 Stunden. Tendenz bei DEGUM hin zu 1,5-, 2-Tageskursen.

Notfallsonographie: G. Mathis berichtet über abgehaltene Kurse und weist auf das Modulsystem der Schweiz hin. Wird gerade aufgebaut, 1 tägige Kurse; zuvor Grundkurs in der Basisnotfallsonographie, weitere Module (Anästhesie, Nephrologie) entstehen dort.

Allgemeinmedizin: K. Somavilla berichtet über Karzinome/Aneurysmen die er im Rahmen von Vorsorgeuntersuchungen entdeckt hat. Dies waren Zufallsbefunde. Der Ultraschall ist im Rahmen einer Vorsorgeuntersuchung nicht vorgesehen, bzw. ist in fast allen Bundesländern keine Verrechnung der Ultraschalluntersuchungen im Rahmen einer Vorsorgeuntersuchung möglich.

Thorax: H. Prosch berichtet, dass der Arbeitskreis Thorax sehr eng an die Not- fallsonographie gebunden ist. $\mathrm{H}$. Prosch schlägt vor, den Arbeitskreis Thorax mit Notfallsonographie zusammenzulegen. H. Prosch berichtet, dass er bzw. Herr Prof. Dr. Peter Zechner abwechselnd Thorax-Kurse veranstaltet haben. Er schlägt vor, diese Kurse nun auch als offizielle ÖGUM-Veranstaltung zu organisieren. G. Mathis schlägt vor, das Thoraxmodul auch mit dem Schweizer Modul zu vergleichen. G. Mathis und H. Prosch werden diesbezüglich weitere Schritte einleiten.

Technik: C. Kollmann berichtet darüber, dass 15-20\% der Schallsonden Probleme aufweisen. Diesbezüglich wurde eine Leitlinie verfasst, wie man mit auftretenden Problemen der Sonden umgehen soll, die auch auf der Website der ÖGUM veröffentlicht wurden.

Kopf/Hals: G. Zettinig macht auf die großen Unterschiede zwischen Deutschland, Österreich und der Schweiz aufmerksam. Darauf basierend wurden Richtlinien erstellt. Das Kurssystem besteht aus einem Grundkurs, danach zwei verschiedene Aufbaukurse: Kopf / Hals und Schilddrüse. Er spricht an, dass niedergelassene Internisten sich mehr für das Zertifikat der ÖÄK interessieren, als für das der ÖGUM.

\section{ÖGUM-Sekretariat}

Neutorgasse 9/2a, AT-1010 Wien

Tel.: + 43 (0)1 5351305

Fax: +43(0)15357037

E-Mail: geschaeftsstelle@oegum.at Öffnungszeiten: Mo-Fr, 9:00-13:00 Uhr Homepage: www.oegum.at

Beiträge/Kursinfo für ÖGUM-Mitteilungen an:

Ass. Prof. Dr. Christian Kollmann

Zentrum f. Medizinische Physik \& Biomedizinische Technik, MedUni Wien Währinger Gürtel 18-20, E 4L

A-1090 Wien

Tel: 0043/1/40400/73730

Fax: 0043/1/40400/3988

E-Mail:

christian.kollmann@meduniwien.ac.at 


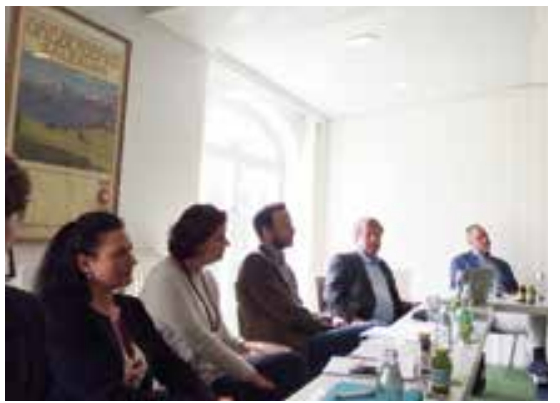

Des Weiteren wurden Programmvorschläge für das DLT in Leipzig eingebracht.

Abdomen: T. Ybinger berichtet über die wenigen Stufe I und Stufe II zertifizierten Ärzte in der ÖGUM. Zertifikat hat eher wenig Bedeutung für Niedergelassene. Interessieren sich eher für das ÖÄK Diplom.

G. Zettinig schlägt vor, die Stufeninhaber auf der Website zu platzieren, um somit für Patienten leichter gefunden zu werden. Stufe II- und III-Inhabern könnte man auch das ÖGUM-Logo zur Verfügung stellen, um dies auf den Websiten der Stufeninhaber zu platzieren, mit direktem Link zur ÖGUM-Website.

Gynäkologie: E. Hafner berichtet über 20 Stufe I- und 10 Stufe II-Anträge, die letztes Jahr bearbeitet wurden. Von 7.-8. Oktober 2016 findet in Seggau der erste Kongress über gynäkologischen Ultraschall statt. Das Stufe II-Treffen letztes Jahr ist bei den Teilnehmer/Innen sehr gut angekommen und wird dieses Jahr wiederholt.

\section{Studententutor}

H. Prosch stellt das Konzept des Studententutors vor. Aufgebaut als Modulsystem, soll in der Studienzeit die erste Hälfte eines Grundkurses bereits absolviert werden. Das erste Modul soll Anatomie und Technik abdecken.

Durchgeführt wird dieser Kurs von Stufe III-Kursleitern, praktische Übungen unter Aufsicht eines von der ÖGUM zertifizierten „Studententutors“. Die zweite Hälfte dann als Ausbildungsarzt. Somit ist die Hälfte eines Grundkurses bereits absolviert, die Attraktivität ist gesteigert an weiteren Kursen teilzunehmen - man wird an die ÖGUM gebunden. Das 16-Stunden Konzept eines Grundkurses ist erfunden worden, als im Studium keine weitere Möglichkeit bestand, einen Ultraschall durchzuführen.

Der Grundkurs ist an sich nicht attraktiv für Ausbildungsärzte, außer man geht nach Deutschland oder in die Schweiz.

G. Mathis begrüßt dieses Konzept sehr und weist u.a. auf das Konzept der Medical School Maribor hin.

Das Konzept wird am Treffen der Vorstände den Schwesterngesellschaften vorgestellt.

\section{Ultraschall 2017}

B. Kasperak stellt die bisherigen Planungen für das Dreiländertreffen in Linz, 11.-13. Oktober 2017, vor. Anwenderseminare werden wie gewohnt am Mitt- woch stattfinden. Ende September 2016 wird eine Aussendung an die Arbeitskreise zur Einreichung der Programmvorschläge mittels Doodle-Umfrage und eines beschreibbaren PDF-Dokumentes durchgeführt. Mitte Jänner folgt die Programmsitzung in Linz, mit den darauf folgenden Sprechereinladungen.

Es war insgesamt wieder eine sehr erfolgreiche Sitzung, wo hinsichtlich Harmonisierung in den Schwesterngesellschaften und Verbesserung der Ultraschallausbildung in Österreich weitere wichtige Weichen vorbereitet wurden. Ich möchte mich herzlich bei allen teilnehmenden Kollegen/Innen für ihren persönlichen Einsatz bedanken.

\section{Mit herzlichem Gruß}

\section{Andrea Klauser | ÖGUM Präsidentin}
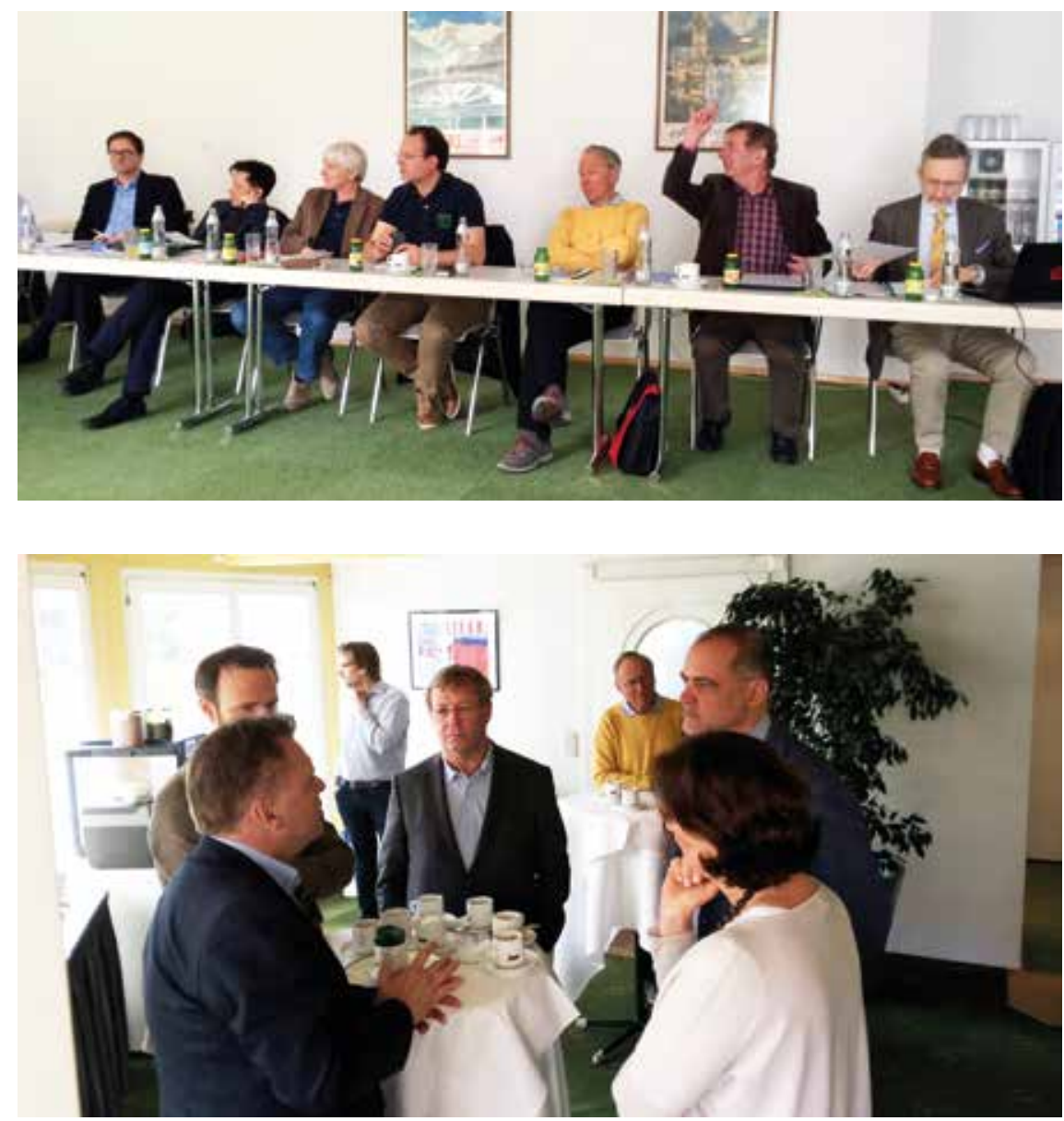